\title{
Recognition of histone acetylation by the GAS41 YEATS domain promotes H2A.Z deposition in non-small cell lung cancer
}

\author{
Chih-Chao Hsu, ${ }^{1,2,8}$ Jiejun Shi, ${ }^{3,8}$ Chao Yuan, ${ }^{1,2,7,8}$ Dan Zhao, ${ }^{4,5,8}$ Shiming Jiang, ${ }^{1,2}$ Jie Lyu, ${ }^{3}$ \\ Xiaolu Wang, ${ }^{1,2}$ Haitao Li, ${ }^{4,5}$ Hong Wen, ${ }^{1,2}$ Wei Li, ${ }^{3}$ and Xiaobing Shi ${ }^{1,2,6}$ \\ ${ }^{1}$ Department of Epigenetics and Molecular Carcinogenesis, The University of Texas MD Anderson Cancer Center, Houston, Texas \\ 77030, USA; ${ }^{2}$ Center for Cancer Epigenetics, The University of Texas MD Anderson Cancer Center, Houston, Texas 77030, USA; \\ ${ }^{3}$ Dan L. Duncan Cancer Center, Department of Molecular and Cellular Biology, Baylor College of Medicine, Houston, Texas 77030, \\ $\mathrm{USA}_{;}{ }^{4} \mathrm{MOE}$ Key Laboratory of Protein Sciences, Beijing Advanced Innovation Center for Structural Biology, Department of Basic \\ Medical Sciences, School of Medicine, Tsinghua University, Beijing 100084, China; ${ }^{5}$ Tsinghua-Peking Joint Center for Life \\ Sciences, Tsinghua University, Beijing 100084, China; ${ }^{6}$ Genetics and Epigenetics Graduate Program, The University of Texas MD \\ Anderson Cancer Center UTHealth Graduate School of Biomedical Sciences, Houston, Texas 77030, USA
}

Histone acetylation is associated with active transcription in eukaryotic cells. It helps to open up the chromatin by neutralizing the positive charge of histone lysine residues and providing binding platforms for "reader" proteins. The bromodomain (BRD) has long been thought to be the sole protein module that recognizes acetylated histones. Recently, we identified the YEATS domain of AF9 (ALL1 fused gene from chromosome 9) as a novel acetyl-lysinebinding module and showed that the ENL (eleven-nineteen leukemia) YEATS domain is an essential acetyl-histone reader in acute myeloid leukemias. The human genome encodes four YEATS domain proteins, including GAS41, a component of chromatin remodelers responsible for H2A.Z deposition onto chromatin; however, the importance of the GAS41 YEATS domain in human cancer remains largely unknown. Here we report that GAS41 is frequently amplified in human non-small cell lung cancer (NSCLC) and is required for cancer cell proliferation, survival, and transformation. Biochemical and crystal structural studies demonstrate that GAS41 binds to histone H3 acetylated on H3K27 and H3K14, a specificity that is distinct from that of AF9 or ENL. ChIP-seq (chromatin immunoprecipitation [ChIP] followed by high-throughput sequencing) analyses in lung cancer cells reveal that GAS41 colocalizes with H3K27 ac and H3K14ac on the promoters of actively transcribed genes. Depletion of GAS41 or disruption of the interaction between its YEATS domain and acetylated histones impairs the association of histone variant H2A.Z with chromatin and consequently suppresses cancer cell growth and survival both in vitro and in vivo. Overall, our study identifies GAS41 as a histone acetylation reader that promotes histone H2A.Z deposition in NSCLC.

[Keywords: GAS41; YEATS domain; histone acetylation; H2A.Z; non-small cell lung cancer]

Supplemental material is available for this article.

Received June 22, 2017; revised version accepted December 19, 2017.

Post-translational modifications (PTMs) on histones play important roles in regulating chromatin dynamics and the accessibility of the underlying DNA in eukaryotes (Strahl and Allis 2000). Acetylation on histone lysine residues is generally associated with active transcription and is modulated by two families of counteracting enzymes: histone acetyltransferases (HATs) and histone deacetylases (HDACs) (Brown et al. 2000; Kouzarides 2007). Addition of an acetyl moiety neutralizes the positive charge on the side chain of lysine residues; in addition, the resulting bulky acetyl group can serve as a docking site for reader proteins, which recognize this specific modification and transduce the molecular signals to elicit various downstream biological outcomes (Jenuwein and Allis 2001). The bromodomain (BRD) has long been thought to be the sole protein module that specifically recognizes acetyl-lysine motifs (Dhalluin et al. 1999), but later studies showed that certain tandem plant homeodomain (PHD) zinc fingers can also bind histone $\mathrm{H} 3$ in an acetylation-

(C) 2018 Hsu et al. This article is distributed exclusively by Cold Spring Harbor Laboratory Press for the first six months after the full-issue publication date (see http://genesdev.cshlp.org/site/misc/terms.xhtml). After six months, it is available under a Creative Commons License (Attribution-NonCommercial 4.0 International), as described at http://creativecommons.org/licenses/by-nc/4.0/. 
sensitive manner (Zeng et al. 2010; Ali et al. 2012; Qiu et al. 2012).

Recently, we identified the YEATS domain of AF9 (ALL1 fused gene from chromosome 9) as a novel reader of histone acetylation (Li et al. 2014). Interestingly, in MLL (mixed-lineage leukemia)-rearranged acute myeloid leukemias (AMLs), ENL (eleven-nineteen leukemia), but not AF9, is essential for AML maintenance (Wan et al. 2017), indicating that the YEATS domain proteins are involved in distinct biological or pathological processes despite the shared biochemical features of their YEATS domains. The YEATS domain has been evolutionarily conserved from yeast to humans (Le Masson et al. 2003). Humans have four YEATS domain-containing proteins, whereas Saccharomyces cerevisiae have three. All YEATS domain proteins interact with chromatin-associated complexes, such as HAT complexes and chromatin remodeling complexes (Schulze et al. 2009); however, the functions of these proteins-and particularly their YEATS domains-are not well understood.

The YEATS domain-containing protein 4 (YEATS4; also known as glioma amplified sequence 41 [GAS41]) is a stoichiometric component of the SRCAP (SNF2-related CREBBP activator protein) and Tip60/p400 chromatin remodeling complexes. In S. cerevisiae, Yaf9, the GAS41 homolog, is associated with both the SWR-1 and NuA4 complexes (Ikeda et al. 1999; Krogan et al. 2003). SWR-1 deposits the histone variant H2A.Z, whereas NuA4 acetylates histones in chromatin (Kobor et al. 2004; Mizuguchi et al. 2004). The Yaf9 YEATS domain was reported to bind to histones $\mathrm{H} 3 / \mathrm{H} 4$ and be required for H2A.Z deposition at gene promoters (Wang et al. 2009). The human SRCAP and Tip60/p400 complexes are the functional equivalents of the yeast SWR1 and NuA4 complexes, respectively. Both SRCAP and Tip60/p400 can exchange the canonical histone H2A for the histone variant H2A. $\mathrm{Z}$ in chromatin (Wong et al. 2007; Cuadrado et al. 2010). Incorporation of H2A.Z and another histone variant, H3.3, results in destabilized nucleosome core particles that are predisposed for disassembly (Jin and Felsenfeld 2007). Therefore, H2A.Z is thought to play an active role in maintaining an accessible chromatin structure (Subramanian et al. 2015). However, the presence of H2A.Z has also been linked to transcriptional repression of some genes (Gevry et al. 2007; Marques et al. 2010; Park et al. 2010; Lee et al. 2012). Nevertheless, although the functions and mechanisms of the SRCAP and Tip60/ p400 complexes are relatively well understood, the role of GAS41 within these complexes remains ill defined. In this study, we characterize the molecular and biological functions of GAS41. We found that GAS41 is frequently amplified in human cancers, including non-small cell lung cancer (NSCLC), and that depletion of GAS41 reduced cancer cell growth, survival, and transformation activity. The YEATS domain of GAS41 bound to acetylated histone H3K27 (H3K27ac) and H3K14 (H3K14ac), which is important for the function of GAS41 in cells. Disruption of the ability of GAS41 to recognize these acetylation marks abrogated global H2A.Z occupancy on chromatin and consequently deactivated target gene expression and suppressed cancer cell growth and survival both in vitro and in a xenograft mouse model. Taken together, our results demonstrate that GAS41 is a histone acetylation reader that controls both $\mathrm{H} 2 \mathrm{~A} . \mathrm{Z}$ dynamics and a transcriptional program essential for NSCLC cell growth and survival.

\section{Results}

GAS41 is amplified in NSCLC and is required for cell growth and survival

GAS41 was originally identified as one of the 12 genes located within chromosomal segment $12 \mathrm{q} 13-15$ that is frequently amplified in glioblastoma (Fischer et al. 1996). To determine whether GAS41 plays a role in human cancers, we first examined GAS41 gene expression across cancers in The Cancer Genome Atlas (TCGA) database via the cBioPortal for Cancer Genomics. Consistent with previous reports (Fischer et al. 1997; Italiano et al. 2008; Persson et al. 2008), GAS41 is amplified in a variety of human cancers, including sarcoma, lung, bladder, and uterine cancers as well as glioblastoma (Fig. 1A). Importantly, GAS41's amplification status is positively correlated with its gene expression levels in these tumors (Supplemental Fig. S1A-E). NSCLC consists of three subtypes: lung adenocarcinoma (LAC), lung squamous cell carcinoma (LSCC), and large cell carcinoma (LCC) (Travis et al. 2013). Comparison of GAS41 gene expression in different NSCLC subtypes in the Oncomine lung cancer data sets revealed that GAS41 is elevated in all NSCLC subtypes compared with normal lung tissues (Fig. 1B; Supplemental Fig. S1F). Therefore, we assessed GAS41 protein levels across a number of NSCLC cell lines. Compared with immortalized "normal" lung fibroblast cell lines (WI-38 and IMR-90) and human bronchial epithelial cells (HBECs) (Ramirez et al. 2004), GAS41 was overexpressed in all NSCLC cell lines that we examined (Fig. 1C). Together, these results suggest that GAS41 is amplified and overexpressed in NSCLC.

As GAS41 protein levels are elevated in cancer cell lines, we wished to determine whether depletion of GAS41 affects lung cancer cell growth and survival. To this end, we knocked down GAS41 gene expression in two lung adenocarcinoma cell lines that express high levels of GAS41, H1299, and H1993 (Fig. 1D) and examined cell proliferation. We observed a marked suppression of cell proliferation in cells treated with GAS41 targeting shRNAs compared with the cells treated with a nontargeting control shRNA (shNT) (Fig. 1E,F). Notably, the extent of growth suppression correlated with the knockdown efficiency: More severe growth defects were observed in the cells with higher knockdown efficiency. We also observed similar growth defects upon GAS41 depletion in several other lung cancer cell lines as well as immortalized HBECs and normal lung fibroblast cells (WI-38 and IMR90) (Supplemental Fig. S1G-M), indicating that the requirement of GAS41 for cell growth is not specific to cancer cells. In clonogenic assays, the GAS41 knockdown cells developed many fewer colonies 

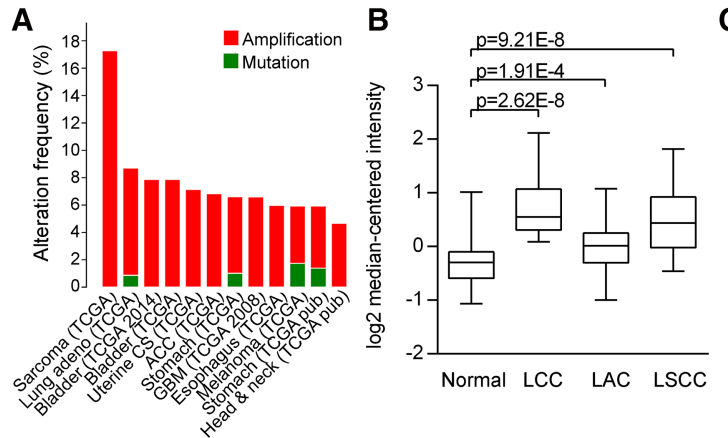

C

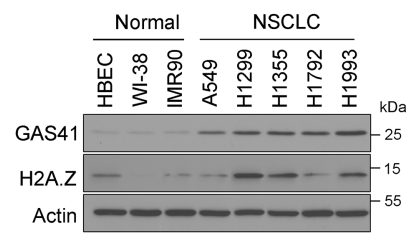

D

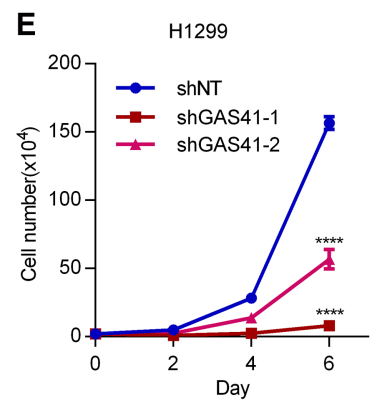

$\mathbf{F}$

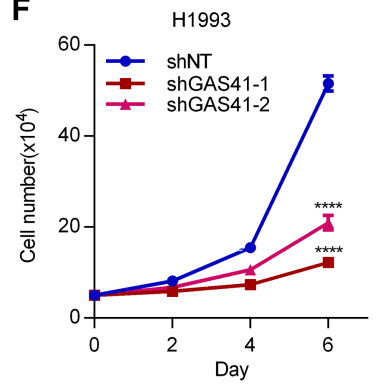

G

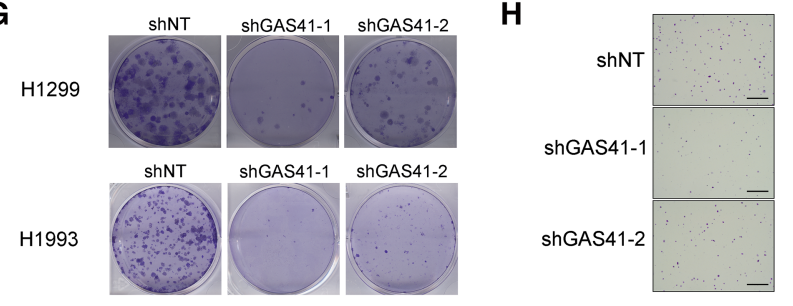

H

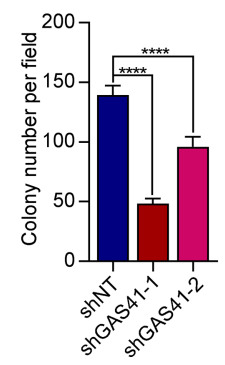

Figure 1. GAS41 is amplified in NSCLC and is required for cancer cell proliferation. (A) GAS41 is frequently amplified in human cancers. Histogram showing the alteration frequency of GAS41. Data were acquired from cBioPortal for Cancer Genomics, focusing on TCGA data sets. (B) GAS41 transcripts are elevated in all NSCLC subtypes. Box and whiskers diagram displaying GAS41 transcript levels. Data were acquired from Oncomine database using the Hou lung data set (Hou et al. 2010). (C) GAS41 is highly expressed in NSCLC. Western blot analysis of GAS41 and H2A.Z expression in immortalized normal lung cells and the indicated NSCLC cell lines. $\beta$-Actin was used as a loading control. $(D)$ Western blot analysis of GAS41 knockdown in H1299 (top panel) and H1993 (bottom panel) cells. Tubulin and $\beta$-actin were used as loading controls. $(E, F)$ Cell proliferation assays of control (shNT) and GAS41 knockdown (shGAS41) in H1299(E) and H1993 (F) cells. Cells (mean \pm SEM; $n=4$ ) were counted for $6 \mathrm{~d}$ after seeding. $\left(^{* * *}\right) P<0.0001$, two-tailed unpaired Student's test. (G) GAS41 is required for cancer cell proliferation. Colony formation assays of cells in $D$. Cells were stained with crystal violet $10 \mathrm{~d}$ after seeding. $(H)$ Loss of GAS41 leads to reduced transformation. Soft agar assay of $\mathrm{H} 1299$ cells as in E. Cell colonies were counted $3 \mathrm{wk}$ after seeding. (Left panel) Representative images. Bar, 1000 um. (Right panel) Quantified results. Error bars represent SEM of six replicates. ${ }^{(* * *)} P$ $<0.0001$, two-tailed unpaired Student's test.

compared with control cells, indicating that GAS41 is also required for lung cancer cell survival (Fig. 1G). Finally, we performed soft agar colony formation assays to determine whether GAS41 knockdown influences anchorage-independent growth, a characteristic of transformed cells (Borowicz et al. 2014). Compared with the control H1299 cells treated with shNT, GAS41 knockdown cells developed fewer colonies in soft agar (Fig. 1H). Taken together, these results indicate that GAS41 is essential for cell growth and survival.

\section{GAS41 and H2A.Z are required for the expression of cell cycle genes}

To determine how GAS41 regulates cancer cell growth and survival, we performed RNA sequencing (RNA-seq) analysis in GAS41 knockdown cells to identify all genes regulated by GAS41. We used GAS41 targeting shRNA\#1 for RNA-seq, since this shRNA exhibited a better knockdown efficiency (Fig. 1D; Supplemental Fig. S2A). By analyzing the RNA-seq data in triplicate, we identified 747 down-regulated genes $\left(\log _{2}\right.$ fold change $\leq-1$; false discovery rate $[\mathrm{FDR}]<0.01)$ and 1504 up-regulated genes $\left(\log _{2}\right.$ fold change $\geq 1$; FDR $<0.01$ ) in GAS41 knockdown cells (Fig. 2A; Supplemental Table S1). Kyoto Encyclopedia of
Genes and Genomes (KEGG) pathway analysis of the differentially expressed genes using DAVID (Database for Annotation, Visualization, and Integrated Discovery) (Huang et al. 2009) revealed that the dysregulated genes were involved in vital biological processes. Down-regulated genes were enriched in pathways regulating DNA replication, cell cycle, and DNA repair pathways, whereas up-regulated genes were enriched in pathways related to extracellular matrix-receptor interaction and cell adhesion (Fig. 2B; Supplemental Table S1). Using quantitative reverse transcription coupled with real-time PCR analysis (qRT-PCR), we validated the RNA-seq data for a subset of genes involved in cell cycle control and DNA replication (Fig. 2C). In all cases, we saw a reduction in expression of the targets selected for RT-qPCR analysis in the GAS41 knockdown cells, with the level of reduction correlating with GAS41 knockdown efficiency. Together, these results suggest that GAS41 is required for the expression of genes involved in critical pathways essential for cell growth and survival.

GAS41 is a stoichiometric component of the SRCAP and Tip60/p400 chromatin remodeling complexes, both of which exchange the canonical histone H2A for the variant histone H2A.Z in chromatin (Wong et al. 2007; Cuadrado et al. 2010). To determine whether GAS41 regulates 
A

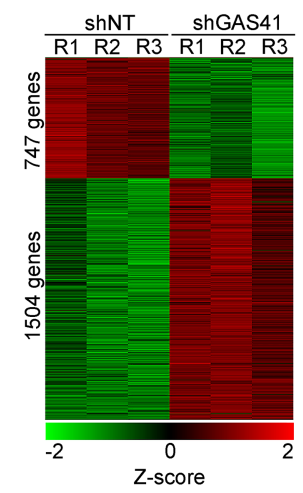

C

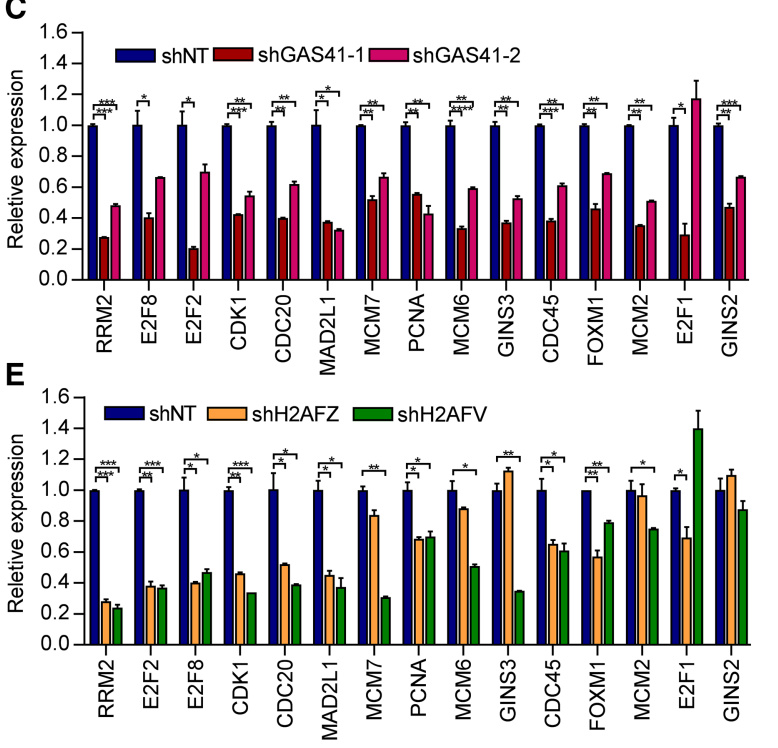

B

\begin{tabular}{lcc} 
GAS41 KD Down Genes & & \\
\hline \multicolumn{1}{c}{ KEGG Pathway } & Count & P Value \\
\hline DNA replication & 10 & $1.57 \mathrm{E}-07$ \\
Cell cycle & 16 & $3.57 \mathrm{E}-07$ \\
Fanconi anemia pathway & 11 & $5.43 \mathrm{E}-07$ \\
Homologous recombination & 6 & $6.59 \mathrm{E}-04$ \\
\hline Pyrimidine metabolism & 10 & $1.08 \mathrm{E}-03$ \\
\hline
\end{tabular}

GAS41 KD Up Genes

\begin{tabular}{lcc}
\hline \multicolumn{1}{c}{ KEGG Pathway } & Count & P Value \\
\hline ECM-receptor interaction & 23 & $6.36 \mathrm{E}-09$ \\
Focal adhesion & 32 & $2.24 \mathrm{E}-06$ \\
PI3K-Akt signaling pathway & 43 & $1.07 \mathrm{E}-05$ \\
Cell adhesion molecules & 24 & $1.39 \mathrm{E}-05$ \\
Cytokine-cytokine receptor interaction & 30 & $1.40 \mathrm{E}-04$ \\
\hline
\end{tabular}

D
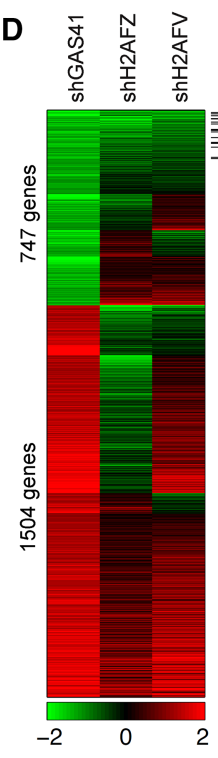

Figure 2. GAS41 regulates the expression of cell cycle-related genes. (A) Heat map display of differentially expressed genes $\left(\log _{2}\right.$ fold change $\geq 1$ ) comparing control (shNT) and GAS41 knockdown (shGAS41) H1299 cells. Data were from three independent RNA-seq experiments. (Red) Up-regulated genes; (green) down-regulated genes in shGAS41 cells compared with shNT cells. (B) KEGG pathway analysis of up-regulated and down-regulated genes in GAS41 knockdown H1299 cells. Data were analyzed using DAVID Bioinformatics Resources 6.8. The most significant five categories are shown. All categories are shown in Supplemental Table S1. (C) qRT-PCR analysis of selected down-regulated genes in GAS41 knockdown (shGAS41) H1299 cells. $\left(^{*}\right) P<0.05$; $\left(^{* *}\right) P<0.01 ;\left(^{* * *}\right) P<0.001 ;(* * * *) P<0.0001$, two-tailed unpaired Student's test. $(D)$ Heat map display of differentially expressed genes in shGAS41, shH2AFZ, and shH2AFV cells comparing control (shNT) H1299 cells. Colors represent $\log _{2}$ fold change of gene expression. (Red) Up-regulated genes; (green) down-regulated genes. (E) qRT-PCR analysis of selected GAS41 targeting cell cycle-related gene expression in control (shNT) and H2A.Z knockdown (shH2AFZ or shH2AFV) H1299 cells. $\left.\left.\left(^{*}\right) P<0.05 ;{ }^{* *}\right) P<0.01 ;{ }^{* * *}\right) P<$ 0.001, two-tailed unpaired Student's test. gene expression through modulation of H2A.Z deposition, we knocked down H2A.Z-encoding genes and compared global gene expression patterns with GAS41 knockdown cells. In mammals, there are two H2A.Z-encoding genes: $H 2 A F V$ and H2AFZ (Subramanian et al. 2015). Although H2AFZ was dominant in controlling global H2A.Z protein levels in $\mathrm{H} 1299$ cells, both genes were essential for cell growth (Supplemental Fig. S2B-D). RNA-seq analysis revealed that, similar to GAS41 knockdown cells, the down-regulated genes in both $H 2 A F V$ knockdown and H2AFZ knockdown cells were strongly enriched in the pathways of cell cycle and DNA replication (Supplemental Fig. S2E; Supplemental Table S1). Unsupervised clustering analysis of dysregulated genes in GAS41, H2AFZ, and $H 2 A F V$ knockdown cells revealed that the majority of GAS41-regulated genes was dysregulated in either $H 2 A F V$ or H2AFZ knockdown cells or both (Fig. 2D). RT-qPCR analysis demonstrated that most GAS41-dependent cell cycle genes were also down-regulated in H2A.Z knockdown cells (Fig. 2E).

\section{GAS41 is required for genome-wide H2A.Z deposition}

Next, we performed chromatin immunoprecipitation (ChIP) experiments followed by high-throughput sequenc- ing (ChIP-seq) to assess the genome-wide occupancy of GAS41 and H2A.Z in H1299 cells. We identified 7365 GAS41-binding peaks and 16,712 H2A.Z peaks (Supplemental Table S2). Notably, GAS41 colocalized with H2A.Z across the genome, with $>80 \%$ of the GAS41 genes being coenriched for H2A.Z (Fig. 3A,B). Because the two GAS41-associated protein complexes SRCAP and Tip60/ p400 are the only chromatin remodelers responsible for H2A.Z deposition onto chromatin, we speculated that depletion of GAS41 would lead to a global reduction of H2A.Z occupancy on chromatin. Indeed, heat map plotting of our H2A.Z ChIP-seq data from control and GAS41 knockdown cells revealed a marked reduction of H2A.Z occupancy at almost all H2A.Z-binding sites (Fig. $3 \mathrm{C})$, suggesting a defect in genome-wide H2A.Z deposition. The reduction of H2A.Z could also be seen at individual GAS41 target genes when visualizing the ChIPseq data with the University of California at Santa Cruz (UCSC) genome browser (Fig. 3D). Finally, we validated the loss of H2A.Z at several of these targets in GAS41 knockdown cells by ChIP-qPCR analysis (Fig. 3E). These results suggest that GAS41 regulates gene expression by modulating global H2A.Z occupancy.

Although GAS41 was detected at only about one-third of H2A.Z-occupied genes, depletion of GAS41 resulted 
Hsu et al.

A

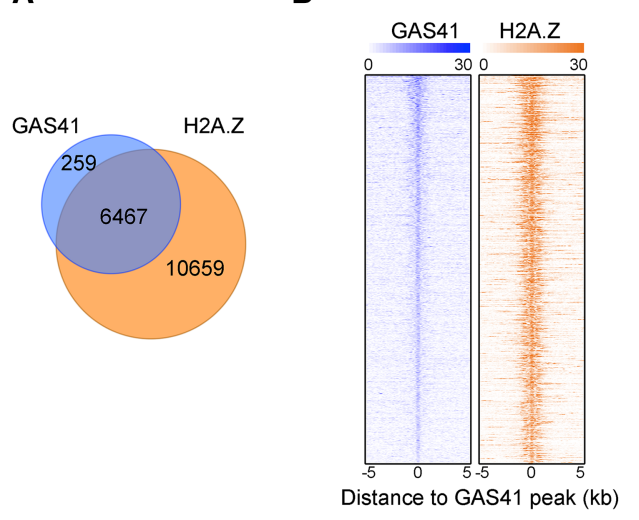

D

E
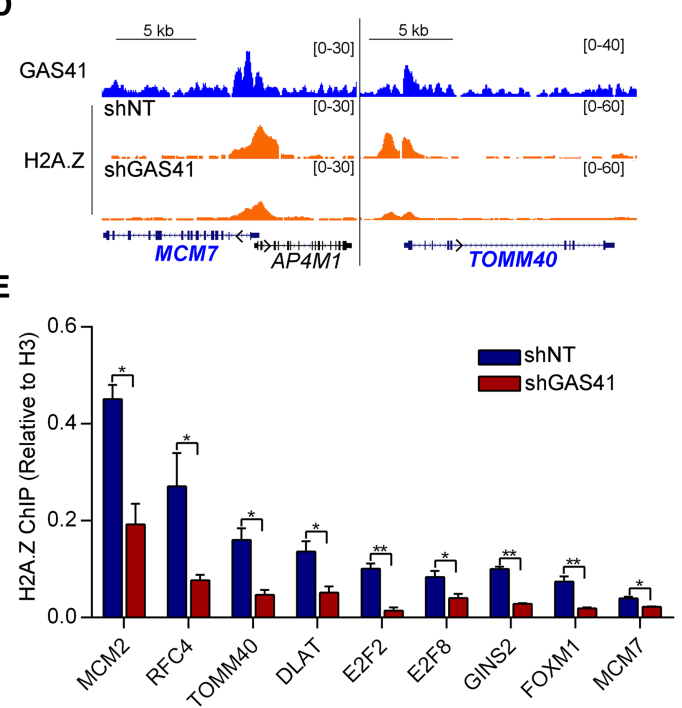

C

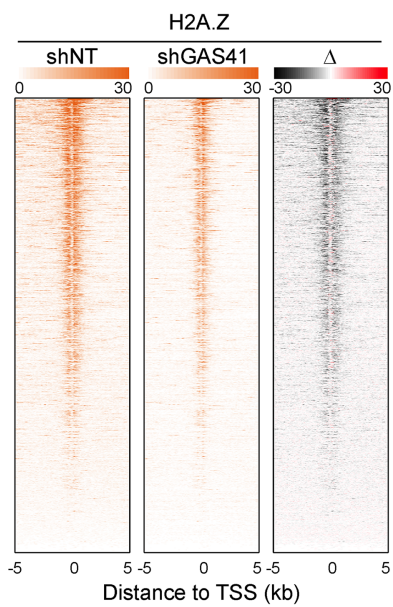

$\mathbf{F}$

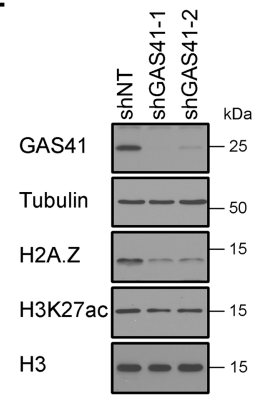

Figure 3. Gas41 is required for genome-wide H2A.Z occupancy in cells. (A) Venn diagram showing the overlap of GAS41- and H2A.Z-occupied genes in H1299 cells. $P=1.21 \times 10^{-9}$ (Super exact test). (B) Heat maps of normalized density of GAS41 and H2A.Z ChIP-seq tags centered on GAS41-binding peaks across a \pm 5 -kb window. The color key represents the signal density, where darker color represents higher ChIP-seq signal. $(C)$ Heat maps of H2A.Z ChIP-seq in control (shNT) and GAS41 knockdown (shGAS41-1) H1299 cells and the difference $(\Delta=$ shGAS41-shNT). (Black) Reduction in shGAS41 cells. $(D)$ Genome browser view of GAS41 (blue) and H2A.Z (orange) peaks. ChIP-seq peaks on the indicated genes in control (shNT) and GAS41 knockdown (shGAS41-1) H1299 cells. (E) ChIP-qPCR of H2A.Z at promoters of the indicated GAS41 target genes in control (shNT) and GAS41 knockdown (shGAS41-1) H1299 cells. Error bars indicate SEM of three repeats. Data are representative of at least two independent experiments. $\left({ }^{*}\right) P<0.05 ;(* *) P<0.01$, two-tailed unpaired Student's test. (F) Western blot analysis of H2A.Z and H3K27 acetylation in control (shNT) and GAS41 knockdown (shGAS41) H1299 cells. Histone H3 and $\beta$-tubulin served as loading controls. in markedly reduced total H2A.Z protein levels in cells (Fig. 3F). This global reduction likely occurred at the protein level, as the expression of H2A.Z-encoding genes remained largely unchanged in GAS41 knockdown cells (Supplemental Fig. S2A). Interestingly, although ectopic expression of H2A.Z was able to restore total H2A.Z protein levels in GAS41 knockdown cells (Supplemental Fig. S3A), it failed to re-establish appropriate H2A.Z occupancy at target gene promoters (Supplemental Fig. S3B) and consequently did not rescue the gene expression and cell proliferation defects of the GAS41 knockdown cells (Supplemental Fig. S3C,D). These results suggest that global reduction of H2A.Z protein levels in GAS41 knockdown cells is a consequence, but not a cause, of defective H2A.Z chromatin deposition.

GAS41 is associated with both the SRCAP and Tip60/ p400 complexes. To further delineate which complex is required for GAS41's functions in H1299 cells, we knocked down SRCAP and EP4OO by specific shRNAs (Supplemental Fig. S3E,F). We found that although EP400 knockdown did not reduce global H2A.Z protein levels as the SRCAP knockdown did, both SRCAP and p400 were required for H2A.Z deposition, expression of GAS41 target genes, and cell proliferation (Supplemental
Fig. S3G-I). Taken altogether, these results indicate that GAS41 promotes H2A.Z deposition through both the SRCAP and Tip60/p400 complexes.

\section{The YEATS domain of GAS41 binds to histone H3K27ac and $\mathrm{H} 3 \mathrm{~K} 14 \mathrm{ac}$}

The recognition of histone $\mathrm{H} 3$ acetylation is an evolutionarily conserved function of the AF9 YEATS domain (Li et al. 2014); thus, we reasoned that the YEATS domain of human GAS41 may also bind to acetylated histones. To test this hypothesis, we performed histone peptide pull-down assays and found that the GAS41 YEATS domain (Fig. 4A) bound specifically to acetylated histone $\mathrm{H} 3$, with strong affinity for H3K27ac and H3K14ac and weak or no binding to other acetylated histone peptides (Fig. 4B). This binding pattern is distinct from that of the AF9 and ENL YEATS domains, which interact preferentially with $\mathrm{H} 3 \mathrm{~K} 9 \mathrm{ac}$ and $\mathrm{H} 3 \mathrm{~K} 27 \mathrm{ac}$, thus indicating that the GAS41 YEATS domain may have a function separate from other YEATS domains.

To decipher the underlying molecular basis for H3K27ac recognition by the GAS41 YEATS domain, we determined the complex structure of the YEATS domain (amino acids 
A

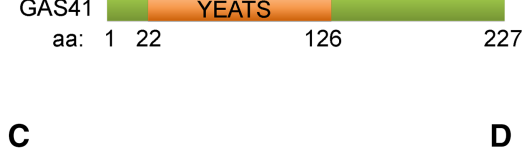

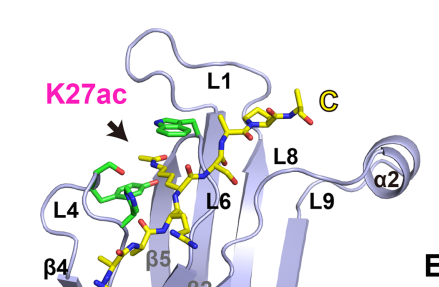

E

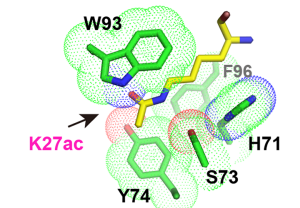

B

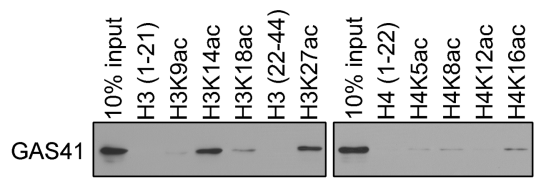

Figure 4. The GAS41 YEATS domain recognizes H3K27ac and H3K14ac. (A) Schematic representation of the GAS41 protein structure. (B) Western blot analysis of histone peptide pull-downs of the GSTGAS41 YEATS domain and the indicated biotinylated peptides. $(C)$ Overall structure of GAS4 $1_{15-159}$ bound to the $\mathrm{H} 3{ }_{15-39} \mathrm{~K} 27 \mathrm{ac}$ peptide. The YEATS domain is shown as a purple ribbon, with key residues of the K27ac-binding pocket depicted as a stick model (green). H3K27ac peptide is shown as yellow sticks. (D) Structural details of H3K27ac readout by a serine-lined aromatic pocket. The key residues in GAS41 YEATS used for K27ac recognition are shown as dots, and the H3K27 peptide is depicted by a yellow stick. $(E)$ Isothermal titration calorimetry (ITC) fitting curves of the Gas41 YEATS domain titrated with the $\mathrm{H} 3_{15-39}$. $\mathrm{K} 27 \mathrm{ac}$ or $\mathrm{H} 3_{1-15} \mathrm{~K} 14 \mathrm{ac}$ peptide. $(F)$ Western blot analysis of peptide pull-downs of the wild-type GAS41 YEATS domain or the indicated point mutants with the unacetylated H3, H3K14ac, and H3K27ac peptides.

15-159) of human GAS41 bound to an $\mathrm{H}_{315-39} \mathrm{~K} 27$ ac peptide at $2.1 \AA$ (the details of crystallization conditions and complex structure will be described in another work). Similar to other YEATS domains (Li et al. 2014; Mi et al. 2017; Wan et al. 2017), the GAS41 YEATS domain adopts an immunoglobin $\beta$-sandwich fold and uses an aromatic sandwich cage for Kac recognition (Fig. 4C). The acetylamide group of $\mathrm{Kac}$ is clamped by the aromatic residues $\mathrm{Y} 74$ and W93 of the GAS41 YEATS domain (Fig. 4D).

Interestingly, among the three H3K27ac binding YEATS domains, we observed two distinct binding modes. The H3K27ac peptide binds in an "N-to-C" orientation to GAS41 and YEATS2 but in an opposite orientation ("C-to-N") to ENL (Supplemental Fig. S4A-C). For ENL binding, the N-terminal "A25-R26" motif of H3K27 contributes to the YEATS domain binding to the acetylated histone peptide. As the "A-R-Kac-S" motif is conserved at both H3K27 and H3K9 (Supplemental Fig. 4D), it explains why ENL shows similar binding affinity to acetylation on these two sites (Wan et al. 2017). In contrast, in the "N-to-C" mode of the H3K27ac peptide binding to GAS41 and YEATS2, the flanking motifs at both sides of H3K27 ("A25-R26" and "S28-A29-P30") contribute to the site-specific recognition of acetylation. The binding of H3P30 to a hydrophobic and flat pocket on the YEATS domains promotes $\mathrm{H} 3 \mathrm{~K} 27 \mathrm{ac}$ recognition. Since such a "Kac-X-X-P" sequence signature is unique to H3K27, both GAS41 and YEATS2 prefer acetylation on H3K27 over H3K9.

Isothermal titration calorimetry (ITC) measured a $K_{\mathrm{D}}$ of $32.7 \mu \mathrm{M}$ for the GAS41 YEATS domain binding to the H3K27ac peptide (Fig. 4E; Supplemental Table S3), an affinity much higher than that of YEATS2 to H3K27ac (226.2 $\mu \mathrm{M})$ (Mi et al. 2017). This is likely attributed to the negatively charged surface of the GAS41 YEATS domain that is favorable for the basic residues on histone H3 (Supplemental Fig. S4A). Interestingly, GAS41 binds to H3K14ac at an even higher affinity $(13.0 \mu \mathrm{M})$ than its binding to H3K27ac (Fig. 4E). Although the molecular basis of $\mathrm{H} 3 \mathrm{~K} 14 \mathrm{ac}$ recognition remains elusive due to the lack of high-quality structure, H3K14ac is likely stabilized by a similar mechanism that involves the two sandwiching aromatic residues (Y74 and W93), as mutation of either residue abolished the binding of the GAS41 YEATS domain to both the H3K27ac and H3K14ac peptides (Fig. 4F).

\section{GAS41 colocalizes with H3K27ac and H3K14ac on active gene promoters}

The in vitro binding and structural data prompted us to determine whether GAS41 colocalizes with acetylated H3K27 and H3K14 in cells. High-throughput sequencing of H3K27ac and H3K14ac ChIP-seq in H1299 cells revealed 16,495 and 11,997 peaks, respectively (Supplemental Table S2). Analysis of all GAS41 ChIP-seq peaks revealed that the majority of GAS41 peaks resided within promoter regions $(91.3 \%)$, and only small fractions were present at either transcribed $(3.1 \%)$ or intergenic $(5.6 \%)$ regions that are possibly enhancers (Fig. 5A). Promoters occupied by GAS41 were highly enriched for acetylated histone $\mathrm{H} 3 ;>90 \%$ of the GAS41-bound genes were co- 
A

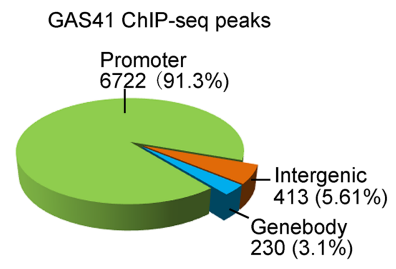

C

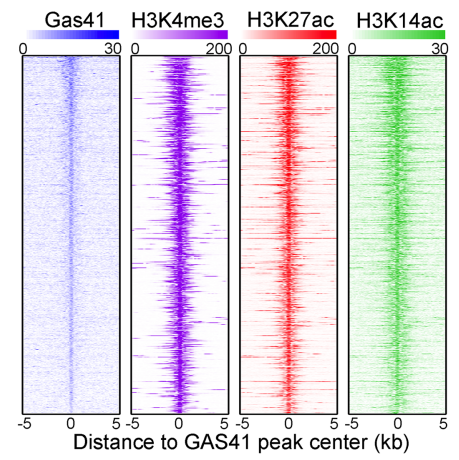

E

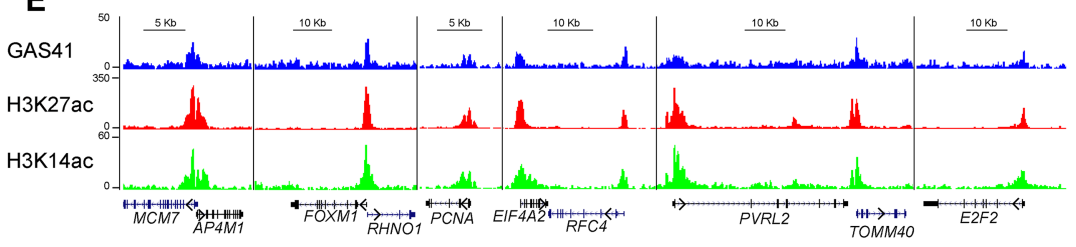

$\mathbf{F}$

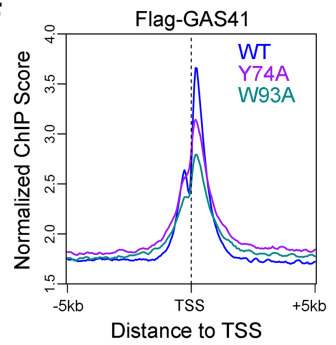

G

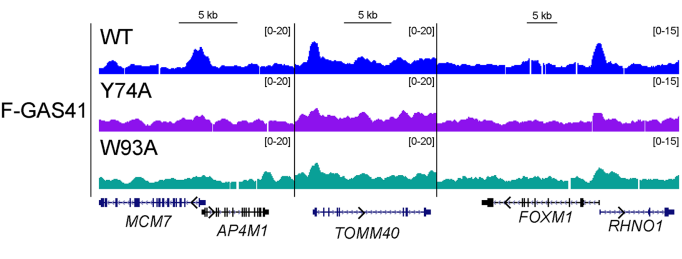

Figure 5. GAS41 colocalizes with H3K14ac and H3K27ac across the genome. (A) Genomic distribution of GAS41 ChIP-seq peaks. The peaks are enriched in the promoter regions (TSS $\pm 3 \mathrm{~kb}) . P=2.2 \times 10^{-16}$, binomial test. $(B)$ Venn diagram showing the overlap of GAS41-, H3K14ac-, and H3K27ac-occupied genes. $P=$ $8.39 \times 10^{-263}$, three-way Fisher's exact test. $(C)$ Heat maps of the normalized density of GAS41, H3K4me3, H3K27ac, and H3K14ac. ChIP-seq tags in H1299 cells centered on GAS41-binding peaks in a $\pm 5-\mathrm{kb}$ window. The color key represents the signal density, where darker color represents higher ChIP-seq signal. (D) Average genome-wide occupancies of GAS41 (blue), H3K27ac (red), and H3K14ac (green) $\pm 5 \mathrm{~kb}$ around the TSS in H1299 cells. (E) Genome browser view of GAS41 (blue), H3K27ac (red), and H3K14ac (green) ChIP-seq peaks on the indicated GAS41-occupied genes in H1299 cells. (F) Average genome-wide occupancies of wild-type Flag-GAS41 (blue) and Y74A (purple) and W93A (cyan) mutants $\pm 5 \mathrm{~kb}$ around the TSS in H1299 cells. $(G)$ Genome browser view of wild-type Flag-GAS41 (blue) and Y74A (purple) and W93A (cyan) mutant ChIP-seq peaks on the indicated GAS41-occupied genes in H1299 cells. occupied by H3K27ac and/or H3K14ac (Fig. 5B). Heat map analysis showed that in addition to H3K27ac and H3K14ac, almost all GAS41 ChIP-seq peaks were enriched in $\mathrm{H} 3 \mathrm{~K} 4 \mathrm{me} 3$, the epigenetic mark for active promoters (Fig. 5C), suggesting that GAS41 may have a positive role in transcription. The average distribution of all GAS41 ChIP-seq peaks across transcription units revealed a strong enrichment at regions within $1 \mathrm{~kb}$ of transcriptional start sites (TSSs) and a strong overlap with the genomic distribution of H3K27ac and H3K14ac (Fig. 5D), a distribution pattern that was reiterated at individual target genes (Fig. 5E).

Next, we asked whether the recognition of $\mathrm{H} 3$ acetylation by the YEATS domain is essential for GAS41 binding on chromatin. To address this question, we established stable cells expressing Flag-tagged wild-type GAS41 or the acetylation-binding-deficient mutants (Y74A and W93A) (Supplemental Fig. S5A) and performed ChIP-seq analysis using the Flag M2 antibody. We found reduced genome-wide occupancy of the Y74A and W93A mutants compared with the wild-type GAS41 (Fig. 5F) as well as their bindings on individual target genes (Fig. 5G), sug- gesting that the YEATS domain is essential for GAS41 binding on chromatin.

The YEATS domain of GAS41 is required for H2A.Z deposition and tumor cell growth

To determine whether the YEATS domain is required for the function of GAS41 in cells, we performed "rescue/reconstitution" experiments by ectopically expressing shRNA-resistant wild-type GAS41 or the acetylationbinding-deficient mutants (Y74A and W93A) in GAS41depleted H1299 cells (Fig. 6A). Again, we observed a reduction in global H2A.Z levels in GAS41 knockdown cells. Importantly, rescue with wild-type GAS41 restored the total levels of H2A.Z in the GAS41 knockdown cells, whereas expression of the acetylation-binding-deficient mutants did not (Fig. 6A). Furthermore, ectopic expression of wildtype GAS41, but not the Y74A and W93A mutants, restored H2A.Z levels at selected target gene promoters to levels comparable with those of control cells (Fig. 6B). The observed defects of the YEATS domain mutants in sustaining normal H2A.Z occupancy at promoters are 


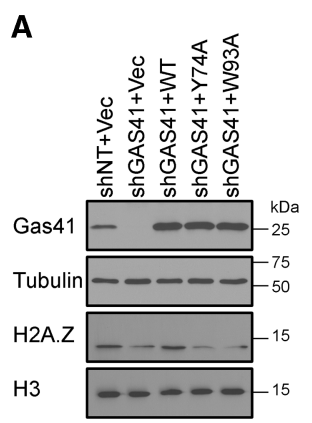

B

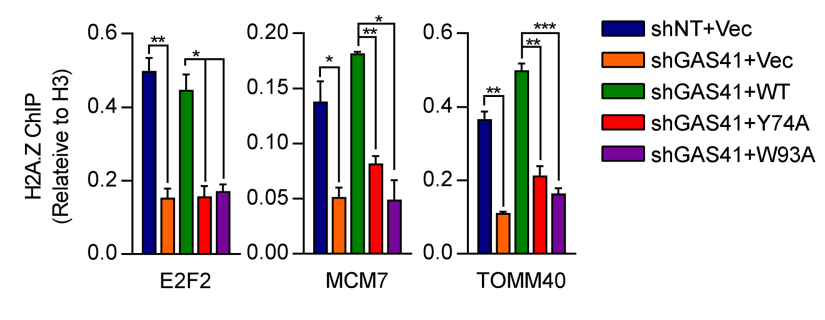

C

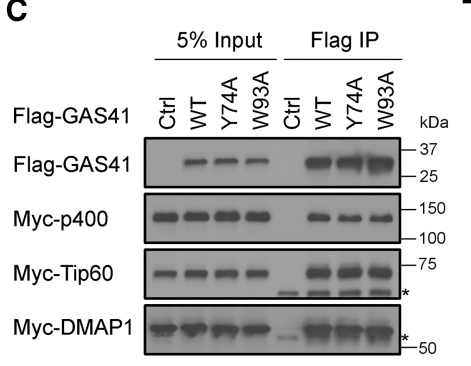

E
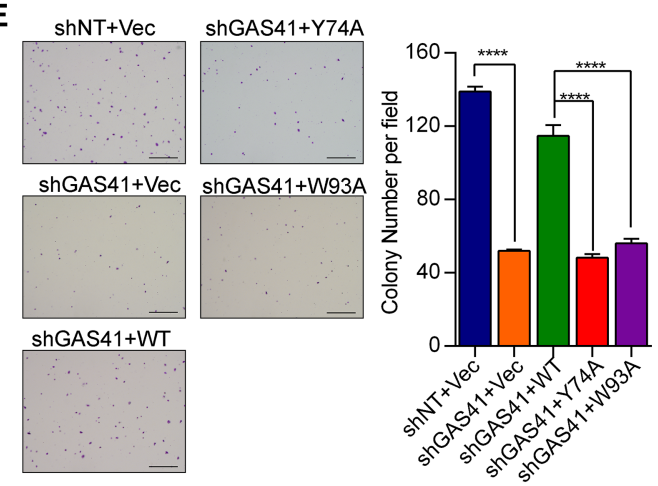

D

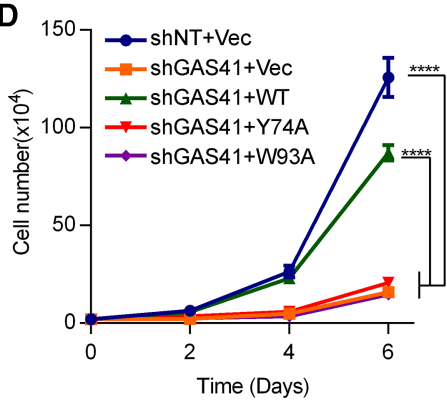

$\mathbf{F}$

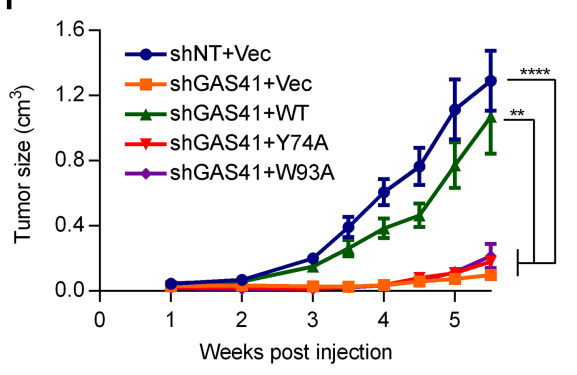

Figure 6. The GAS41 YEATS domain is required for H2A.Z deposition and tumor growth. (A) Western blot analysis of GAS41 and H2A.Z protein levels in shGAS41-1-expressing H1299 cells rescued with wild-type GAS41 or the indicated mutants. (B) ChIP-qPCR analysis of H2A.Z promoter occupancy of the indicated GAS41occupied genes in shGAS41-1-expressing H1299 cells rescued with wild-type or mutant GAS41. $\left(^{*}\right) P<0.05$; $\left(^{* *}\right) P<0.01 ;(* * *)$ $P<0.001$, two-tailed unpaired Student's test. $(C)$ Coimmunoprecipitation analysis of the interaction of Flag-GAS41 and the Myc-tagged EP400 N-terminal fragment, Tip60, and DMAP1. Asterisks indicate IgG heavy chain. $(D)$ Cell proliferation assay of shGAS41-1-expressing H1299 cells rescued with wild-type GAS41 or the indicated mutants. (E) Soft agar assay of shGAS41-1-expressing H1299 cells rescued with wildtype GAS41 or the indicated mutants. Bar, $1000 \mu \mathrm{m}$. (Left panels) Representative images. (Right panel) Quantified results. $\left.{ }^{* * * *}\right) P$ $<0.0001$, two-tailed unpaired Student's test. $(F)$ Volumes of tumors resulting from H1229 cells (as in A) subcutaneously transplanted into immunodeficient nude mice. Mean \pm SEM. $n=9-10$. Tumors were monitored for $5.5 \mathrm{wk} .(* *) P<0.01 ;(* * *) P<$ 0.0001, two-tailed unpaired Student's test. likely due to the deficiency of GAS41 in recognizing histone acetylation, as the protein-protein interactions of GAS41 with other complex components were not affected by the mutations (Fig. 6C). Taken together, these results indicate that the YEATS domain of GAS41 is required for SRCAP and/or p400 complex-dependent maintenance of H2A.Z levels on target gene promoters.

Next, we sought to determine whether the YEATS domain is required for GAS41 function in regulating cell growth and survival. We performed cell proliferation and soft agar colony formation assays using the above-mentioned reconstitution system. We found that ectopic expression of wild-type GAS41 restored cell proliferation and anchorage-independent cell growth in the GAS41-depleted cells, but the Y74A and W93A mutants did not (Fig. $6 \mathrm{D}, \mathrm{E})$. Finally, to determine whether GAS41 is important for tumor growth in a mouse xenograft model, we examined the proliferation of control H2199 cells, GAS41 knockdown cells, and the reconstituted cells injected into immunodeficient nude mice. We found that depletion of GAS41 suppressed tumor growth in mice. Importantly, wild-type GAS41, but not the H3 acetylationbinding-deficient mutants (Y74A and W93A), led to tumor growth of the GAS41 knockdown cells in mice (Fig. 6F; Supplemental Fig. S5B). Taken together, our results suggest a model in which GAS41 recognizes acetylated histone H3 and recruits the SRCAP and Tip60/p400 complexes to target gene promoters, which in turn deposit H2A.Z onto chromatin to promote expression of the genes essential for cancer cell proliferation and survival (Fig. 7).

\section{Discussion}

For more than a decade, BRDs have been recognized as the principle readers of histone acetylation and are important for mediating signaling at chromatin to regulate transcription (Sanchez and Zhou 2009). Research from our laboratory has shown that the YEATS domain is also a reader of acetylation that impacts transcription. The YEATS domain of AF9 links histone acetylation to DOT1L-dependent histone H3K79 methylation during transcriptional elongation (Li et al. 2014), and the ENL YEATS domain is essential for the function of the superelongation complex in the maintenance of AML (Wan et al. 2017). In addition to AF9 and ENL, the human genome encodes two 


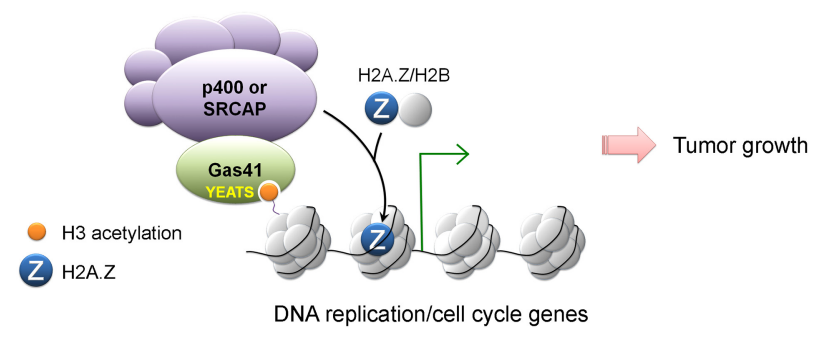

Figure 7. Working model. GAS41 binds to acetylation on H3K27 and H3K14 through its YEATS domain and facilitates the recruitment of the Tip60/p400 and/or SRCAP complex to gene promoters. The Tip60/p400 or SRCAP complex in turn exchanges the canonical H2A for H2A.Z at gene promoter regions, thus promoting the expression of target genes that are essential for cell proliferation and survival.

other YEATS domain proteins, YEATS2 and YEATS4/ GAS41, both of which are components of histone-modifying or chromatin remodeling complexes (Cai et al. 2003, 2005; Suganuma et al. 2008; Wang et al. 2008).

Despite the fact that AF9 and ENL recognize acetylation on H3K9, H3K18, and H3K27, whereas YEATS2 prefers H3K27ac, all three YEATS domains share a similar mechanism for recognizing acetylation of lysine within the "RK" motif (Li et al. 2014; Zhao et al. 2016; Wan et al. 2017). The YEATS domain of GAS41 also uses aromatic residues conserved among all YEATS domains, forming a Ser/Thr-lined sandwiching cage for encapsulation of the acetyl moiety. However, in addition to recognizing histone H3K27ac, GAS41 also binds strongly to $\mathrm{H} 3 \mathrm{~K} 14 \mathrm{ac}$, a residue that lacks an "RK" motif. We were not able to crystallize GAS41 in complex with H3K14ac, but it will be interesting to determine in future studies how GAS41 binds to both H3K27ac and H3K14ac with strong affinities. Nevertheless, together, our results demonstrated that recognition of acetylation on histone $\mathrm{H} 3$ is a common feature of all YEATS domains involved in diverse processes regulating chromatin and transcription.

In addition to the GAS41 YEATS domain, other H3K14ac-specific readers have been reported, such as the double PHD fingers of DPF3b and MOZ (Zeng et al. 2010; Ali et al. 2012; Qiu et al. 2012) and the ZMYND8 BRD (Li et al. 2016). The specificity of these acetylation readers depends on their adjacent PHD fingers that bind to the N-terminal H3K4 region of the same histone tail and thus physically limit accessibility to other acetylated lysines. In contrast, the GAS41 YEATS domain is unique in that it is a standalone reader that can discriminate acetylation on $\mathrm{H} 3 \mathrm{~K} 14$ or H3K9. Both H3K14ac and H3K9ac are present predominately at the promoters of actively transcribed genes (Li et al. 2007a) and are usually deposited by the GCN5/PCAF-containing HAT complexes (Nagy et al. 2010; Spedale et al. 2012; Wang and Dent 2014). It is not clear why the YEATS domain of GAS41 specifically recognizes H3K14ac, whereas those of AF9 and ENL prefer H3K9ac. The Tora laboratory (Karmodiya et al. 2012) has shown previously that in mouse embryonic stem cells, H3K14ac not only shares occupancy with H3K9ac at ac- tive promoters but also is enriched at a subset of inactive promoters, suggesting that $\mathrm{H} 3 \mathrm{~K} 14 \mathrm{ac}$ may prime inactive genes for stimulus-dependent activation. It remains to be determined whether GAS41 also occupies the promoters of these "poised" genes in mouse embryonic stem cells to help regulate stimulus-responsive gene expression.

$\mathrm{H} 3 \mathrm{~K} 27 \mathrm{ac}$ is an epigenetic mark of active enhancers. Biochemically, GAS41 binds to H3K27ac and H3K14ac equally well; however, in cells, GAS41 is enriched at promoter rather than enhancer regions. As the majority GAS41 protein moieties resides in SRCAP or Tip60/p400 complexes, it is likely that other reader modules within these complexes contribute to GAS41-binding specificity in cells. In line with this speculation, we previously identified the PHD finger of the complex subunit ING3 as a reader of H3K4me3 (Shi et al. 2006), which is highly enriched at active promoters, and the chromodomain domain of MRG15 has been shown to bind to H3K36me3 (Li et al. 2007b), which is also associated with actively transcribed genes. Therefore, GAS41 likely cooperates with ING3, MRG15, and possibly other undefined readers to form a "reader module" within the SRCAP or Tip60/p400 complex to facilitate the recruitment of the complex to chromatin. In addition, GAS41 associates with some DNAbinding transcription factors that likely play a role in determining the primary localization of GAS41. For example, it has been shown that GAS41 directly interacts with Myc, which resides in the proximal promoter regions (Piccinni et al. 2011). In line with these results, Ingenuity Pathway Analysis (IPA) of our GAS41 ChIP-seq data reveals MYCN as one of the upstream regulators of GAS41 (Supplemental Table S2).

Previous studies have revealed that GAS41 is amplified in several human cancers, such as astrocytoma (Fischer et al. 1997), liposarcoma (Italiano et al. 2008; Persson et al. 2008), and NSCLC (Pikor et al. 2013). Part of its tumor-promoting activity is thought to be mediated through negative regulation of the p53 pathway. For example, GAS41 physically interacts with p53 and regulates p53 protein stability independently of the SRCAP or Tip60/ p400 complex (Park et al. 2011). In addition, GAS41 is present at the promoters of p53-regulated genes, such as p21, and suppresses gene expression (Park and Roeder 2006; Pikor et al. 2013). The suppression of $\mathrm{p} 21$ gene expression is dependent on the SRCAP or Tip60/p400 complex, as eviction of $\mathrm{H} 2 \mathrm{~A}$. $\mathrm{Z}$ is a prerequisite for $\mathrm{p} 21$ gene activation in response to DNA damage or the onset of replicative senescence (Gevry et al. 2007; Marques et al. 2010; Park et al. 2010; Lee et al. 2012). In our studies, depletion of GAS41 suppressed cell growth and survival of both p53 wild-type (e.g., A549) and mutant (e.g. H1299 and H1993) NSCLC cell lines, suggesting that GAS41 regulates the expression of a plethora of genes beyond the p53-p21 axis. Indeed, IPA of both GAS41 ChIP-seq and RNA-seq data in the p53-null H1299 cells reveals enrichment of many pathways, including cell cycle, DNA replication, and cell death and survival.

Regulation of target gene expression by GAS41 is likely mediated through H2A.Z, as depletion of GAS41 leads to both gene-specific and global reduction of H2A.Z 
occupancy, which is important because H2A.Z has been shown to possess an oncogenic role regulating similar sets of genes in different types of tumors. For example, H2A.Z is both overexpressed in and enriched at promoters of cell cycle regulatory genes in bladder cancer (Kim et al. 2013), liver cancer (Yang et al. 2016), and melanoma (Vardabasso et al. 2015). Importantly, although H2A.Z is enriched at both active and poised gene promoters, acetylated H2A.Z is associated exclusively with gene activation (Ku et al. 2012; Valdes-Mora et al. 2012). As recognition of $\mathrm{H} 3$ acetylation by the GAS41 YEATS domain is essential for the function of GAS41 in chromatin occupancy of H2A.Z and gene expression, it would be interesting to determine whether GAS41 can also recognize acetylation on H2A.Z.

In summary, we identified GAS41 as a histone acetylation reader and a candidate oncogene that is amplified in NSCLC. Given that targeting the BRD histone acetylation readers holds great promise for treating a variety of human cancers (Fujisawa and Filippakopoulos 2017), the YEATS family of histone acetylation readers may also provide attractive therapeutic targets for the treatment of this disease.

\section{Materials and methods}

ChIP and ChIP-seq analysis

ChIP analysis was performed essentially as described (Wen et al. 2014). Briefly, cells were cross-linked with $1 \%$ formaldehyde for $10 \mathrm{~min}$, and cross-linking was stopped with $125 \mathrm{mM}$ glycine. The isolated nuclei were resuspended in nucleus lysis buffer and sonicated using a Bioruptor Sonicator (Diagenode). The samples were immunoprecipitated with $2-4 \mu \mathrm{g}$ of the appropriate antibodies overnight at $4^{\circ} \mathrm{C}$. Protein $\mathrm{A} / \mathrm{G}$ beads were added and incubated for $1 \mathrm{~h}$, and the immunoprecipitates were washed twice each with low-salt, high-salt, and LiCl buffers. Eluted DNA was reverse cross-linked, purified using a PCR purification kit (Qiagen), and analyzed by quantitative real-time PCR on the ABI 7500-FAST system using the Power SYBR Green PCR master mix (Applied Biosystems). Statistical differences were calculated using a two-way unpaired Student's $t$-test. The primer sequences are listed in Supplemental Table S4.

For ChIP-seq, ChIP experiments were performed as described above. Samples were sequenced using the Illumina Solexa HiSeq 2500. The raw reads were mapped to human reference genome NCBI 37 (hg19) using the Solexa data processing pipeline, allowing up to two mismatches. The genome ChIP-seq profiles were generated using MACS 1.4.2 (Zhang et al. 2008) with only uniquely mapped reads. Clonal reads were automatically removed by MACS. The ChIP-seq profiles were normalized to $10,000,000$ total tag numbers, and peaks were called at $P$-values $\leq 1 \times 10^{-8}$. The ChIP-seq read density was determined by deepTools and then visualized by using $\mathrm{R}$.

RNA extraction, reverse transcription, and real-time PCR analysis

Total RNA was prepared using an RNeasy plus kit (Qiagen) and reverse-transcribed using an iScrip reverse transcription kit (Bio-Rad). qPCR analyses were performed on an ABI 7500FAST sequence detection system using the Power SYBR Green PCR master mix (Applied Biosystems). Gene expression was calculated following normalization to GAPDH levels using the comparative $\mathrm{Ct}$ (cycle threshold) method. Experimental data are presented as the mean \pm standard deviation of the mean unless stated otherwise. Statistical significance was calculated using a two-tailed unpaired Student's $t$-test on two experimental conditions, with $P<0.05$ considered statistically significant. The primer sequences for qPCR are listed in Supplemental Table S4.

RNA-seq was performed using an Illumina HiSeq 2000. Three biological replicates were prepared for each condition. Sequencing reads were mapped to the human genome (hg19) using TopHat (version 2.0.10). The overall mapping rate was $95 \%-$ $99 \%$. The number of fragments in each known gene from the RefSeq database (downloaded from UCSC genome browser on June 2, 2014) was enumerated using htseq-count, part of the HTSeq package (version 0.6.0) (http://www-huber.embl.de/ users/anders/HTSeq). The differential expression between conditions was statistically assessed with $\mathrm{R} /$ Bioconductor package edg$\mathrm{eR}$ (version 3.8.6). Genes with a FDR of $\leq 0.01$ and a fold change of $\geq 2$ were called in GAS41 knockdown cells, and genes with a FDR of $\leq 0.05$ and a fold change of $\geq 1.5$ were called in $H 2 A F Z$ and $H 2 A F V$ knockdown cells. Hierarchical clustering was performed using the hclust function in $\mathrm{R}$ using expression values estimated by edgeR. The expression values for each gene across samples were centered by their medians and scaled by standard deviation before clustering. Euclidean distance and the ward.D2 clustering method were used. The heat map was plotted using the pheatmap function in R.

Statistical analyses

Experimental data are presented as the mean \pm the standard deviation of the mean unless stated otherwise. Statistical significance was calculated using a two-tailed unpaired $t$-test on two experimental conditions, with $P<0.05$ considered statistically significant unless stated otherwise. Statistical significance levels are denoted as follows: $P<0.05(*), P<0.01(* *), P<0.001\left({ }^{* * *}\right)$, and $P<0.0001\left(^{* * * *}\right)$. No statistical methods were used to predetermine sample size. The statistical significance of the Venn diagram data was calculated using SuperExactTest in R (https ://cran.r-project.org/package=SuperExactTest).

The methods for protein production, peptide pull-downs, ITC, cell culture, viral transduction, RNAi, cell proliferation, colony formation assays, soft agar colony formation, and xenografts in nude mice are described in the Supplemental Material.

\section{Accession numbers}

The ChIP-seq and RNA-seq data have been deposited in the Gene Expression Omnibus database with accession number GSE100347.

\section{Acknowledgments}

We thank J. Heymach, J. Minna, J. Kurie, M. Bedford, M.G. Lee, and J. Huang for sharing reagents. We thank B. Dennehey for editing the manuscript and scientific discussion. We thank the MD Anderson Science Park Next-Generation Sequencing Facility (Cancer Prevention Research Institute of Texas [CPRIT] RP170002) for Solexa sequencing. This work was supported in part by grants from the National Key R\&D Program of China (2016YFA0500700) and the National Science Foundation for Distinguished Young Scholars of China (31725014) to H.L.; CPRIT (RP150292) and the National Institutes of Health (NIH; R01HG007538 and R01CA193466) to W.L.; NIH/National 
Cancer Institute (NCI; 1R01CA204020), CPRIT (RP160237 and RP160739), the Welch Foundation (G1719), and the Texas Tobacco Settlement to X.S.; the National Natural Science Foundation of China (81602476) to C.Y.; and the National Post-doctoral Program for Innovative Talents (BX201600088) to D.Z. D.Z. is a postdoctoral fellow of the Tsinghua-Peking Joint Center for Life Sciences. X.S. is a Leukemia and Lymphoma Society Career Development Program Scholar.

Author contributions: X.S., W.L., and H.L. conceived the study; C.-C.H. and C.Y. performed the biochemical and cellular studies; J.S. performed the bioinformatics analysis; D.Z. performed the structural studies; S.J. performed xenograft assays, X.W. provided technical assistance; and X.S., H.L., and C.-C.H. wrote the paper with comments from H.W., J.S., and W.L. X.S. is a Scientific Advisory Board member of EpiCypher.

\section{References}

Ali M, Yan KZ, Lalonde ME, Degerny C, Rothbart SB, Strahl BD, Cote J, Yang XI, Kutateladze TG. 2012. Tandem PHD fingers of MORF/MOZ acetyltransferases display selectivity for acetylated histone $\mathrm{H} 3$ and are required for the association with chromatin. J Mol Biol 424: 328-338.

Borowicz S, Van Scoyk M, Avasarala S, Karuppusamy Rathinam MK, Tauler J, Bikkavilli RK, Winn RA. 2014. The soft agar colony formation assay. J Vis Exp e51998.

Brown CE, Lechner T, Howe L, Workman JL. 2000. The many HATs of transcription coactivators. Trends Biochem Sci 25: 15-19.

Cai Y, Jin J, Tomomori-Sato C, Sato S, Sorokina I, Parmely TJ, Conaway RC, Conaway JW. 2003. Identification of new subunits of the multiprotein mammalian TRRAP/TIP60-containing histone acetyltransferase complex. I Biol Chem 278: 42733-42736.

Cai Y, Jin J, Florens L, Swanson SK, Kusch T, Li B, Workman JL, Washburn MP, Conaway RC, Conaway JW. 2005. The mammalian YL1 protein is a shared subunit of the TRRAP/TIP60 histone acetyltransferase and SRCAP complexes. I Biol Chem 280: 13665-13670.

Cuadrado A, Corrado N, Perdiguero E, Lafarga V, Munoz-Canoves P, Nebreda AR. 2010. Essential role of p18Hamlet/SRCAPmediated histone H2A.Z chromatin incorporation in muscle differentiation. EMBO I 29: 2014-2025.

Dhalluin C, Carlson JE, Zeng L, He C, Aggarwal AK, Zhou MM. 1999. Structure and ligand of a histone acetyltransferase bromodomain. Nature 399: 491-496.

Fischer U, Meltzer P, Meese E. 1996. Twelve amplified and expressed genes localized in a single domain in glioma. Hum Genet 98: 625-628.

Fischer U, Heckel D, Michel A, Janka M, Hulsebos T, Meese E. 1997. Cloning of a novel transcription factor-like gene amplified in human glioma including astrocytoma grade I. Hum Mol Genet 6: 1817-1822.

Fujisawa T, Filippakopoulos P. 2017. Functions of bromodomaincontaining proteins and their roles in homeostasis and cancer. Nat Rev Mol Cell Biol 18: 246-262.

Gevry N, Chan HM, Laflamme L, Livingston DM, Gaudreau L. 2007. p21 transcription is regulated by differential localization of histone H2A.Z. Genes Dev 21: 1869-1881.

Hou J, Aerts J, den Hamer B, van Ijcken W, den Bakker M, Riegman $\mathrm{P}$, van der Leest C, van der Spek P, Foekens JA, Hoogsteden HC, et al. 2010. Gene expression-based classification of non-small cell lung carcinomas and survival prediction. PLoS One 5: e10312.
Huang DW, Sherman BT, Lempicki RA. 2009. Systematic and integrative analysis of large gene lists using DAVID bioinformatics resources. Nat Protoc 4: 44-57.

Ikeda K, Steger DJ, Eberharter A, Workman JL. 1999. Activation domain-specific and general transcription stimulation by native histone acetyltransferase complexes. Mol Cell Biol 19: 855-863.

Italiano A, Bianchini L, Keslair F, Bonnafous S, Cardot-Leccia N, Coindre JM, Dumollard JM, Hofman P, Leroux A, Mainguene C, et al. 2008. HMGA2 is the partner of MDM2 in well-differentiated and dedifferentiated liposarcomas whereas CDK4 belongs to a distinct inconsistent amplicon. Int I Cancer 122: 2233-2241.

Jenuwein T, Allis CD. 2001. Translating the histone code. Science 293: 1074-1080.

Jin C, Felsenfeld G. 2007. Nucleosome stability mediated by histone variants H3.3 and H2A.Z. Genes Dev 21: 1519-1529.

Karmodiya K, Krebs AR, Oulad-Abdelghani M, Kimura H, Tora L. 2012. H3K9 and H3K14 acetylation co-occur at many gene regulatory elements, while $\mathrm{H} 3 \mathrm{~K} 14 \mathrm{ac}$ marks a subset of inactive inducible promoters in mouse embryonic stem cells. BMC Genomics 13: 424.

Kim K, Punj V, Choi J, Heo K, Kim JM, Laird PW, An W. 2013. Gene dysregulation by histone variant H2A.Z in bladder cancer. Epigenetics Chromatin 6: 34.

Kobor MS, Venkatasubrahmanyam S, Meneghini MD, Gin JW, Jennings JL, Link AJ, Madhani HD, Rine J. 2004. A protein complex containing the conserved Swi2/Snf2-related ATPase Swrlp deposits histone variant H2A.Z into euchromatin. PLOS Biol 2: E131.

Kouzarides T. 2007. Chromatin modifications and their function. Cell 128: 693-705.

Krogan NJ, Keogh MC, Datta N, Sawa C, Ryan OW, Ding H, Haw RA, Pootoolal J, Tong A, Canadien V, et al. 2003. A Snf2 family ATPase complex required for recruitment of the histone H2A variant Htz1. Mol Cell 12: 1565-1576.

Ku MC, Jaffe JD, Koche RP, Rheinbay E, Endoh M, Koseki H, Carr SA, Bernstein BE. 2012. H2A.Z landscapes and dual modifications in pluripotent and multipotent stem cells underlie complex genome regulatory functions. Genome Biol 13: R85.

Lee K, Lau ZZ, Meredith C, Park JH. 2012. Decrease of p400 ATPase complex and loss of H2A.Z within the p21 promoter occur in senescent IMR-90 human fibroblasts. Mech Ageing Dev 133: 686-694.

Le Masson I, Yu DY, Jensen K, Chevalier A, Courbeyrette R, Boulard Y, Smith MM, Mann C. 2003. Yaf9, a novel NuA4 histone acetyltransferase subunit, is required for the cellular response to spindle stress in yeast. Mol Cell Biol 23: 60866102.

Li B, Carey M, Workman JL. 2007a. The role of chromatin during transcription. Cell 128: 707-719.

Li B, Gogol M, Carey M, Lee D, Seidel C, Workman JL. 2007b. Combined action of PHD and chromo domains directs the Rpd3S HDAC to transcribed chromatin. Science 316: 10501054.

Li Y, Wen H, Xi Y, Tanaka K, Wang H, Peng D, Ren Y, Jin Q, Dent SY, Li W, et al. 2014. AF9 YEATS domain links histone acetylation to DOT1L-mediated H3K79 methylation. Cell 159: 558-571.

Li N, Li YY, Lv J, Zheng XD, Wen H, Shen HJ, Zhu GJ, Chen TY, Dhar SS, Kan PY, et al. 2016. ZMYND8 reads the dual histone mark H3K4me1-H3K14ac to antagonize the expression of metastasis-linked genes. Mol Cell 63: 470-484. 
Marques M, Laflamme L, Gervais AL, Gaudreau L. 2010. Reconciling the positive and negative roles of histone H2A.Z in gene transcription. Epigenetics 5: 267-272.

Mi W, Guan H, Lyu J, Zhao D, Xi Y, Jiang S, Andrews FH, Wang X, Gagea M, Wen $H$, et al. 2017. YEATS2 links histone acetylation to tumorigenesis of non-small cell lung cancer. Nat Commun 8: 1088.

Mizuguchi G, Shen X, Landry J, Wu WH, Sen S, Wu C. 2004. ATP-driven exchange of histone $\mathrm{H} 2 \mathrm{AZ}$ variant catalyzed by SWR1 chromatin remodeling complex. Science 303: 343348.

Nagy Z, Riss A, Fujiyama S, Krebs A, Orpinell M, Jansen P, Cohen A, Stunnenberg HG, Kato S, Tora L. 2010. The metazoan ATAC and SAGA coactivator HAT complexes regulate different sets of inducible target genes. Cell Mol Life Sci 67: 611-628.

Park JH, Roeder RG. 2006. GAS41 is required for repression of the p53 tumor suppressor pathway during normal cellular proliferation. Mol Cell Biol 26: 4006-4016.

Park JH, Sun XJ, Roeder RG. 2010. The SANT domain of p400 ATPase represses acetyltransferase activity and coactivator function of TIP60 in basal p21 gene expression. Mol Cell Biol 30: 2750-2761.

Park JH, Smith RJ, Shieh SY, Roeder RG. 2011. The GAS41PP2C $\beta$ complex dephosphorylates p53 at serine 366 and regulates its stability. J Biol Chem 286: 10911-10917.

Persson F, Olofsson A, Sjogren H, Chebbo N, Nilsson B, Stenman G, Aman P. 2008. Characterization of the 12q amplicons by high-resolution, oligonucleotide array CGH and expression analyses of a novel liposarcoma cell line. Cancer Lett 260: 37-47.

Piccinni E, Chelstowska A, Hanus J, Widlak P, Loreti S, Tata AM, Augusti-Tocco G, Bianchi MM, Negri R. 2011. Direct interaction of Gas41 and Myc encoded by amplified genes in nervous system tumours. Acta Biochim Pol 58: 529-534.

Pikor LA, Lockwood WW, Thu KL, Vucic EA, Chari R, Gazdar AF, Lam S, Lam WL. 2013. YEATS4 is a novel oncogene amplified in non-small cell lung cancer that regulates the p53 pathway. Cancer Res 73: 7301-7312.

Qiu Y, Liu L, Zhao C, Han CC, Li FD, Zhang JH, Wang Y, Li GH, Mei YD, Wu MA, et al. 2012. Combinatorial readout of unmodified H3R 2 and acetylated H3K14 by the tandem PHD finger of MOZ reveals a regulatory mechanism for HOXA9 transcription. Genes Dev 26: 1376-1391.

Ramirez RD, Sheridan S, Girard L, Sato M, Kim Y, Pollack J, Peyton M, Zou Y, Kurie JM, Dimaio JM, et al. 2004. Immortalization of human bronchial epithelial cells in the absence of viral oncoproteins. Cancer Res 64: 9027-9034.

Sanchez R, Zhou MM. 2009. The role of human bromodomains in chromatin biology and gene transcription. Curr Opin Drug Discov Devel 12: 659-665.

Schulze JM, Wang AY, Kobor MS. 2009. YEATS domain proteins: a diverse family with many links to chromatin modification and transcription. Biochem Cell Biol 87: 65-75.

Shi X, Hong T, Walter KL, Ewalt M, Michishita E, Hung T, Carney D, Pena P, Lan F, Kaadige MR, et al. 2006. ING2 PHD domain links histone $\mathrm{H} 3$ lysine 4 methylation to active gene repression. Nature 442: 96-99.

Spedale G, Timmers HT, Pijnappel WW. 2012. ATAC-king the complexity of SAGA during evolution. Genes Dev 26: $527-541$.
Strahl BD, Allis CD. 2000. The language of covalent histone modifications. Nature 403: 41-45.

Subramanian V, Fields PA, Boyer LA. 2015. H2A.Z: a molecular rheostat for transcriptional control. F1000prime Rep 7: 01 .

Suganuma T, Gutierrez JL, Li B, Florens L, Swanson SK, Washburn MP, Abmayr SM, Workman JL. 2008. ATAC is a double histone acetyltransferase complex that stimulates nucleosome sliding. Nat Struct Mol Biol 15: 364-372.

Travis WD, Brambilla E, Riely GJ. 2013. New pathologic classification of lung cancer: relevance for clinical practice and clinical trials. J Clin Oncol 31: 992-1001.

Valdes-Mora F, Song IZ, Statham AL, Strbenac D, Robinson MD, Nair SS, Patterson KI, Tremethick DJ, Stirzaker C, Clark SJ. 2012. Acetylation of H2A.Z is a key epigenetic modification associated with gene deregulation and epigenetic remodeling in cancer. Genome Res 22: 307-321.

Vardabasso C, Gaspar-Maia A, Hasson D, Punzeler S, Valle-Garcia D, Straub T, Keilhauer EC, Strub T, Dong J, Panda T, et al. 2015. Histone variant H2A.Z.2 mediates proliferation and drug sensitivity of malignant melanoma. Mol Cell 59: 75-88.

Wan L, Wen H, Li Y, Lyu J, Xi Y, Hoshii T, Joseph JK, Wang X, Loh YE, Erb MA, et al. 2017. ENL links histone acetylation to oncogenic gene expression in acute myeloid leukaemia. Nature 543: 265-269.

Wang L, Dent SY. 2014. Functions of SAGA in development and disease. Epigenomics 6: 329-339.

Wang YL, Faiola F, Xu M, Pan S, Martinez E. 2008. Human ATAC Is a GCN5/PCAF-containing acetylase complex with a novel NC2-like histone fold module that interacts with the TATA-binding protein. J Biol Chem 283: 33808-33815.

Wang AY, Schulze JM, Skordalakes E, Gin JW, Berger JM, Rine J, Kobor MS. 2009. Asf1-like structure of the conserved Yaf9 YEATS domain and role in H2A. Z deposition and acetylation. Proc Natl Acad Sci 106: 21573-21578.

Wen H, Li Y, Xi Y, Jiang S, Stratton S, Peng D, Tanaka K, Ren Y, Xia Z, Wu J, et al. 2014. ZMYND11 links histone $\mathrm{H} 3.3 \mathrm{~K} 36 \mathrm{me} 3$ to transcription elongation and tumour suppression. Nature 508: 263-268.

Wong MM, Cox LK, Chrivia JC. 2007. The chromatin remodeling protein, SRCAP, is critical for deposition of the histone variant H2A.Z at promoters. I Biol Chem 282: 26132-26139.

Yang HD, Kim PJ, Eun JW, Shen Q, Kim HS, Shin WC, Ahn YM, Park WS, Lee JY, Nam SW. 2016. Oncogenic potential of histone-variant H2A.Z.1 and its regulatory role in cell cycle and epithelial-mesenchymal transition in liver cancer. Oncotarget 7: 11412-11423.

Zeng L, Zhang Q, Li S, Plotnikov AN, Walsh MJ, Zhou MM. 2010. Mechanism and regulation of acetylated histone binding by the tandem PHD finger of DPF3b. Nature 466: 258-262.

Zhang Y, Liu T, Meyer CA, Eeckhoute J, Johnson DS, Bernstein BE, Nusbaum C, Myers RM, Brown M, Li W, et al. 2008. Model-based analysis of ChIP-seq (MACS). Genome Biol 9: R137.

Zhao D, Guan H, Zhao S, Mi W, Wen H, Li Y, Zhao Y, Allis CD, Shi X, Li H. 2016. YEATS2 is a selective histone crotonylation reader. Cell Res 26: 629-632. 


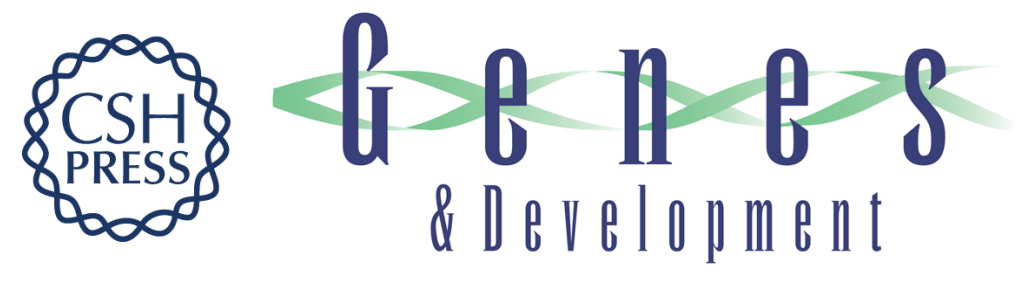

\section{Recognition of histone acetylation by the GAS41 YEATS domain promotes H2A.Z deposition in non-small cell lung cancer}

Chih-Chao Hsu, Jiejun Shi, Chao Yuan, et al.

Genes Dev. 2018, 32: originally published online February 1, 2018

Access the most recent version at doi:10.1101/gad.303784.117

\section{Supplemental http://genesdev.cshlp.org/content/suppl/2018/02/01/gad.303784.117.DC1 Material}

References This article cites 60 articles, 22 of which can be accessed free at: http://genesdev.cshlp.org/content/32/1/58.full.html\#ref-list-1

Creative This article is distributed exclusively by Cold Spring Harbor Laboratory Press for the first Commons six months after the full-issue publication date (see

License http://genesdev.cshlp.org/site/misc/terms.xhtml). After six months, it is available under a Creative Commons License (Attribution-NonCommercial 4.0 International), as described at http://creativecommons.org/licenses/by-nc/4.0/.

Email Alerting Receive free email alerts when new articles cite this article - sign up in the box at the top Service right corner of the article or click here.

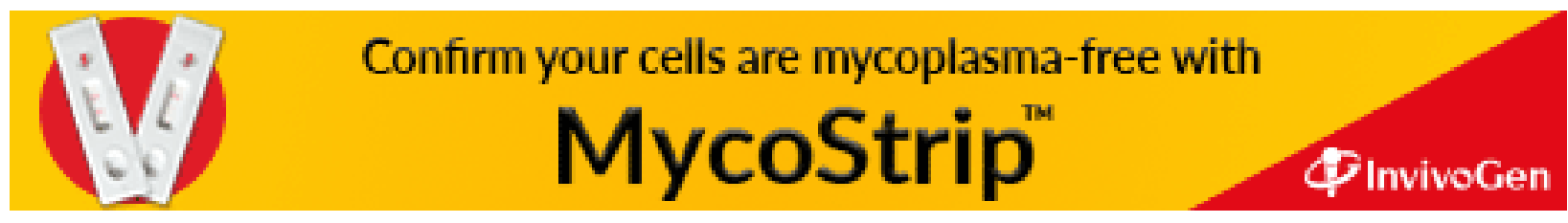

